\title{
A New Method to Identify the Sixteen Coefficients of Hydrodynamic Bearings Used in Disk Drive Spindle Motor
}

\author{
J.H. Wang and W.L. Hwang \\ Sound and Vibration Laboratory, Department of Power Mechanical Engineering, \\ National Tsing Hua University, Hsinchu, Taiwan
}

(Received 24 October 2003; accepted 4 February 2004)

\begin{abstract}
The spindle motor is an important component used in most disk media devices. Nowadays, the spindle is generally supported by two journal bearings with a herringbone-grooved structure. Because the two bearings generally are not identical, there are sixteen bearing coefficients to be identified. In this work, two new identification methods are proposed. The basic concept of the two methods is the same, but there is a slight difference between them. The proposed methods use the measured transfer functions to identify the parameters. Because measurement noise is unavoidable, the derivation of the proposed methods has taken the noise effect into special consideration. The simulation results show that the identification accuracy of the proposed methods is far better than that of an existing method, which also has been especially developed to identify the sixteen coefficients of two bearings. ${ }^{11}$
\end{abstract}

\section{INTRODUCTION}

The spindle motor is an important component of a computer disk drive. The spindle of the motor is generally supported with ball bearings, hydrodynamic bearings or sintered porous bearings. However, the percentage of spindle motors equipped with hydrodynamic bearings has significantly increased recently. This is due to the fact that spindle motors with hydrodynamic bearings offer better dynamic behaviours, i.e., lower non-repeated run-out and lower noise levels, than that of spindle motors with ball bearings or sintered porous bearings. In the past, several technical difficulties have limited the application of hydrodynamic bearings in spindle motors. One knows that spindle motors generally are used in a vertical direction. Consequently, the bearings possess no static preload in the radial direction. One knows that a rotor with plain fluid bearings without static preload generally is dynamically unstable. ${ }^{1}$ The second difficulty is the bearing seal. A standard hydrodynamic bearing generally is equipped with an oil supply and cooling system to replenish the oil leaked from both sides of the bearing and to take the heat away from the bearing. Cost considerations and space limitation do not allow the hydrodynamic bearings used in spindle motor to have an oil supply and cooling system. The above two main difficulties have been solved recently with different methods. For instance, instead of a plain surface, a herringbone-grooved structure is widely used to increase the stability of the spindle motor, as shown in Fig. 1. The seal problem was solved with different oil reservoirs or even with a magneto-fluid as a seal element. In the past, different computational methods have been proposed to calculate the dynamic properties of plain fluid bearings. ${ }^{2-4}$ However, the calculated results generally suffer from significant error due to different simplifications in the theoretical model. The dynamic properties of a bearing with a herringbone-grooved structure are more difficult to calculate with a theoretical method than those of a hydrodynamic bearing with a plain surface. This is due to the fact that the herringbone-grooved structure is generally very complicated, and consequently, very difficult to model accurately by a theoretical method. For instance, the nominal diameter of bearings used in a spindle motor of a disk drive is about $3 \mathrm{~mm}$, and the maximum depth and maximum width of the 3-D herringbone-groove are only $2 \mu \mathrm{m}$ and $10 \mu \mathrm{m}$, respectively.

Due to the theoretical modelling difficulty, the experimental method to identify the dynamic coefficients of the herringbone-grooved bearings, becomes very important. In the past, several different identification methods in the timedomain $^{5,6}$ or the frequency domain ${ }^{7-9}$ were developed to estimate the dynamic coefficients of hydrodynamic bearings. However, the above-mentioned methods can only be applied to identify the eight bearing coefficients of a rotor with two identical bearings. If a rotor is supported by two hydrodynamic bearings which are not identical, then there are sixteen bearing coefficients to be identified. The two hydrodynamic bearings used in the spindle motor generally are not identical. The main reason for this is the manufacturing tolerance. As mentioned, the nominal diameter of a typical bearing used in a spindle motor is about $3 \mathrm{~mm}$, and the nominal bearing clearance (i.e., the difference between the bearing and spindle diameters) is $0.003 \mathrm{~mm}$. If the manufacturing tolerance of the bearing diameter is only $\pm 0.5 \mu \mathrm{m}$, then the possible variation of bearing clearance will be higher than $30 \%$. Besides, the structure of the herring bone groove is very fine, and the manufacture variation of the groove structure has a significant effect on the dynamic characteristics of the bearings. Consequently, the parameters of the two bearings are different, and there are sixteen bearing parameters to be identified.

Lee and Hong proposed a method which theoretically could be used to identify the sixteen bearing parameters. ${ }^{10}$ However, the method was based on an important assumption that the backward vibration could be measured when the rotor was excited by an imbalance. So far, no report has been 